\title{
An Intelligent Group Decision Support System and Its Application for Project Performance Evaluation
}

\author{
Fahimeh Ramezani, Jie Lu \\ Decision Systems \& e-Service Intelligence Lab \\ Centre for Quantum Computation \& Intelligent Systems \\ School of Software, Faculty of Engineering and Information Technology \\ University of Technology, Sydney, PO Box 123, Broadway NSW 2007 Australia
}

Fahimeh.Ramezani@student.uts.edu.au,Jie.Lu@uts.edu.au

\begin{abstract}
:
Purpose - In any organization there are main goals, with lots of projects designed to achieve these goals. It is important for any organization to determine how much these projects affect the achievement of these goals. The purpose of this study is to develop a Fuzzy Multiple Attribute-based Group Decision Support System (FMAGDSS) to evaluate projects' performance in promoting the organization's goals utilizing Simple Additive Weighting (SAW) algorithm and Technique for Order of Preference by Similarity to Ideal Solution (TOPSIS) algorithm. The proposed FMAGDSS deals with choosing the most appropriate fuzzy ranking algorithm for solving a given Fuzzy Multi Attribute Decision Making (FMADM) problem with both qualitative and quantitative criteria (attributes), and uncertain judgments of decision makers.

Design/methodology/approach - In this paper, a Fuzzy Multi Attribute Group Decision Support System (FMAGDSS) model is designed to determine scores and ranks of every project in promoting the organization's goals. In the first step of FMAGDSS model, all projects are assessed by experts based on evaluation criteria and the organization's goals. The proposed FMAGDSS model will then choose the most appropriate fuzzy ranking method to solve the given Fuzzy Multi Attribute Decision Making (FMADM) problem. Finally, a sensitivity analysis system is developed to assess the reliability of the decision making process and provide an opportunity to analyze the impacts of "criteria weights" and "projects' performance" on evaluating projects in achieving the organizations' goals, and to assess the reliability of the decision making process. In addition, a software prototype has been developed on the basis of FMAGDSS model that can be applied to solve every FMADM problem that needs to rank alternatives according to certain attributes.

Findings - The result of this study simplifies and accelerates the evaluation process. The proposed system not only helps organizations to choose the most efficient projects for sustainable development, but also helps them to assess the reliability of the decision making process, and decrease the uncertainty in final decisin caused by uncertain judgment of decision makers.

Research limitations/implications - Future studies are suggested to expand this system to evaluate and rank the project proposals. To achieve this goal, the efficiency of the projects in line with organization's goals, should be predicted.

Practical implications - The proposed FMAGDSS model has been implemented into a software tool for projects' performance evaluation. This software is dynamic to the numbers and the types of criteria (qualitative and quantitative), the number of alternatives and decision makers, and decision making subjects, so it is applicable for a large variety of decision making environments.

Originality/value - This study contributes to the relevant literature by proposing a FMAGDSS model to evaluate projects in promoting organization's goals. The proposed FMAGDSS has ability to choose the most appropriate fuzzy ranking algorithm to solve a given FMADM problem based on the type and the number of attributes and alternatives, considering the least computation and time consumption for ranking alternatives.
\end{abstract}

Keywords Decision support systems, Fuzzy sets, Multi attribute decision making, Project evaluation

\section{Introduction}

Projects ranking and determining the most effective projects to achieve organization goals is a complex problem that requires considering of conditions from multiple perspectives and including the decision makers in the planning process. The necessity to take into account several decision parameters, apart from purely economic ones, such as socio-political, technical, institutional and environmental, lead to the use of multi-criteria decision methods. Moreover, most of the decisions are taken in a group environment (Goletsis et al., 2003, Zhang and Lu, 2003, Lu et al., 2007, Lu et al., 2009, Ma et al., 2010).

Dey (2006) proposed a decision support system that analyses projects with respect to market, technicalities, and social and environmental impact in an integrated framework using an analytic hierarchy process (AHP), and a Multiple-Attribute Decision-Making (MADM) technique. Liang et al. (2006) developed a model to evaluate generation projects by comprehensive utilization of fuzzy appraisal and an analytic hierarchy process (AHP). Sanna et al. (2008) proposed the creation of a hierarchy between the different projects based on ratings expressed as fuzzy numbers. Imoto et al. (2008) employed a principal component model, dual scaling, AHP and fuzzy regression analysis to analyze the results of experts' evaluation in selecting submitted proposals for R\&D and to model the experts' evaluation. 
Chiang and Che (2010) also applied the fuzzy AHP and fuzzy data envelopment analysis (DEA) to develop an evaluation and ranking methodology, to assist decision makers to select NPD projects with development potential and high added value. These works applied the AHP method, which restricts the decision maker (DM) to pair-wise comparison, but in most situations DMs are interested in evaluating attributes independently. Henriksen and Palocsay (2008) proposed an excel-base, user-friendly decision support system for evaluating and ranking R\&D projects. Buyukozkan and Ruan (2008) presented an evaluation model based on the fuzzy multi-criteria decision making (MCDM) method to measure the performance of software development projects. For this purpose they applied and extended a compromise ranking method (known as the VIKOR method) that was proposed to identify compromised solutions, by providing a maximum group utility for the majority and a minimum of an individual regret for the opponent. Marques et al. (2011) proposed a new multi-dimensional Project Performance Measurement System that would enable managers to deal with the volume of data. Lu et al (2011) proposed a new model to evaluate the proposal of a garment manufacturing New Product Development (NPD) under the theme of well-being design. In addition they developed the FHCGDM method and a specialized software tool for garment NPD evaluation to directly support garment NPD evaluations and the establishment of a corresponding relationship between human-sense and machine measurements. Baykasoglu et al. (2011) proposed a practical fuzzy rating and ranking approach to prioritize project activities with fuzzy attributes. Saghaei and Didehkhani (2011) designed an adaptive neuro fuzzy inference system that is capable of considering interrelations between criteria, and then applied a fuzzy weighted additive goal programming model, to propose a comprehensive methodology for evaluation and selection of the six sigma projects. Mao and Wu (2011) applied fuzzy mathematics to assess the levels of income risk and cost risk in real estate investment, and then adjusted the relevant parameters of a fuzzy real option, based on the above risk assessment of the real estate project, to improve assessment of engineering project risk and initial investment decision-making.

However, there are many researches in this field, none of them considered choosing the most appropriate fuzzy ranking methods for solving their given MADM problem. In addition, in previous works the proposed methodology is applicable for a particular FMADM problem, and the reliability of the final decisions have not been assessed. This research proposes a new Fuzzy Multiple Attribute-based Group Decision Support System (FMAGDSS) model, which is applicable for solving every Fuzzy Multi Attribute Decision Making (FMADM) problem that needs to rank alternatives according to certain attributes, with the ability to select the most appropriate fuzzy ranking algorithm to solve a given FMADM problem. In addition, it contains a sensitivity analysis system, which provides an opportunity to assesses the reliability of the decision making process.

This paper is organized as follows. Section 2 explains fuzzy ranking algorithms. In Section 3, we describe the method for choosing the most suitable fuzzy ranking algorithm to solve given FMADM problems. In Section 4 the sensitivity analysis methods are presented. In Section 5 the proposed FMAGDSS conceptual model for project evaluation is described. The proposed new algorithm to evaluate projects is provided in Section 6. Finally, Section 7 describes the software prototype that has been developed to simulate designed FMAGDSS model to solve project evaluation problems.

\section{The Main Fuzzy Ranking Algorithms}

Two fuzzy ranking algorithms: (1) SAW and (2) Chen and Hwang fuzzy ranking algorithms (Chen et al., 1992), are chosen to solve a FMADM problem for evaluating projects. These algorithms can be applied to solve both MADM and FMADM problems with both crisp and fuzzy numbers. Moreover, despite algorithms such as AHP, these two algorithms give DMs an opportunity to assess attributes individually. 


\subsection{Simple Additive Weighting Algorithm (SAW)}

The SAW algorithm is known as the weighted sum algorithm and is probably the best known and most widely used MADM method (Hwang and Yoon, 1981). The basic logic of SAW is to obtain a weighted sum of the performance ratings of each alternative over all attributes (Fishburn, 1967, MacCrimmon, 1968). With a normalized decision matrix $\left(r_{i j}\right)$ and a weight vector $\left(w_{j}\right)$, the overall preference value of each alternative $\left(U_{i}\right)$ is obtained by:

$$
U_{i}=\sum_{j=1}^{n} w_{j} \cdot r_{i j}
$$

The greater the value $\left(U_{i}\right)$ the more preferred the alternative $\left(A_{i}\right)$. Research results have shown that the linear form of trade-offs between attributes used by the SAW algorithm produces extremely close approximations to complicated nonlinear forms, while being far easier to use and understand (Hwang and Yoon, 1981).

This algorithm is appropriate for solving problems with triangle or trapezoidal fuzzy numbers; otherwise, other algorithms are more appropriate (Chen et al., 1992).

\subsection{Chen and Hwang fuzzy ranking algorithm for solving MADM problems}

Considering fuzzy approaches for solving MADM problems, Chen and Hwang (1992) realized that all approaches have two defects: (1) Most of them have the problem of cumbersome computations. As a result, none is suitable for solving problems with more than ten alternatives associated with more than ten attributes. This drawback certainly limits their applicability to real world problems. (2) Some of these approaches require that the elements in the impact matrix be presented in a fuzzy format, even though they are crisp in nature.

In FMADM methods, qualitative numbers convert to fuzzy numbers. Bass and Kwakernak (1977), Bonissone (1982), Chen (1988), Efstathiou and Rajkovic (1979), Efstathiou and Tong (1982), Kerre (1982) and Wenstop (1967) have proposed scales for converting qualitative numbers to fuzzy numbers, and then applied the FMADM method to solve MADM problems. Chen and Hwang (1992) identified a new scale with a combination of previous scales and proposed a new approach to solve FMADM problems that is less complex and needs less computation. For instance, they claim that applying their scale solves a $50 * 50$ matrix as simply as a $5 * 5$ matrix. In addition, MADM problems can be meaningfully and efficiently solved in a fuzzy environment. The basic assumption of the Chen and Hwang approach is that the MADM problem may contain fuzzy and crisp data. This FMADM approach is composed of two phases. Phase one consists of two major steps:

1) Linguistic-term conversion, which transforms the impact value into a fuzzy set if they are verbal terms.

2) Conversion from a fuzzy set to a crisp value set where all the fuzzy sets are assigned crisp scores. The results of this phase produce a new impact matrix that only contains numeric data.

In the second phase, a classical MADM method can be utilized to determine the ranking order of alternatives. In following sections, the procedure of fuzzy impact transformation, and the introduction of Chen and Hwang algorithm for solving MADM problems are described.

\subsubsection{Linguistic-term conversion}

A numerical approximation system is proposed to systematically transform linguistic terms to their corresponding fuzzy set. The transformation requires eight conversion scales, as shown in Figure 1. 
The conversion scales are proposed by synthesizing and modifying the work of Baas and Kwakernak (1977), and Bonissone (1982). In the procedure of the linguistic-term conversion, the principle is to simply select a scale figure that contains all the verbal terms given by the DM and use the membership function set for that figure to represent the meaning of the verbal terms.

\subsubsection{Conversion from a fuzzy set to a crisp value}

The second step of a fuzzy impact transformation is to convert the fuzzy set to crisp scores. In this section, a modified L-R scoring approach based on Jain's (1976, 1977) and Chen's (1985) work is introduced. The crisp score of a fuzzy set $M$ is obtained as follows. Assume a maximizing fuzzy set and a minimizing fuzzy set, which are defined as:

$$
\begin{aligned}
& \mu_{\text {max }}(x)= \begin{cases}x, & 0 \leq x \leq 1 \\
0, & \text { otherwise }\end{cases} \\
& \mu_{\text {min }}(x)= \begin{cases}1-x, & 0 \leq x \leq 1 \\
0, & \text { otherwise }\end{cases}
\end{aligned}
$$

The right score of $\mathrm{M}$ can be determined using:

$$
\mu_{R}(M)=\sup _{x}\left\lfloor\mu_{M}(x) \wedge \mu_{\max }(x)\right\rfloor
$$

Similarly, the left score of $\mathrm{M}$ can be determined using:

$$
\mu_{L}(M)=\sup \left\lfloor\mu_{M}(x) \wedge \mu_{\text {min }}(x)\right\rfloor
$$

Given the left and right scores of $M$, the total score of $M$ can be calculated using:

$$
\mu_{T}(M)=\left[\mu_{R}(M)+1-\mu_{L}(M)\right] / 2
$$
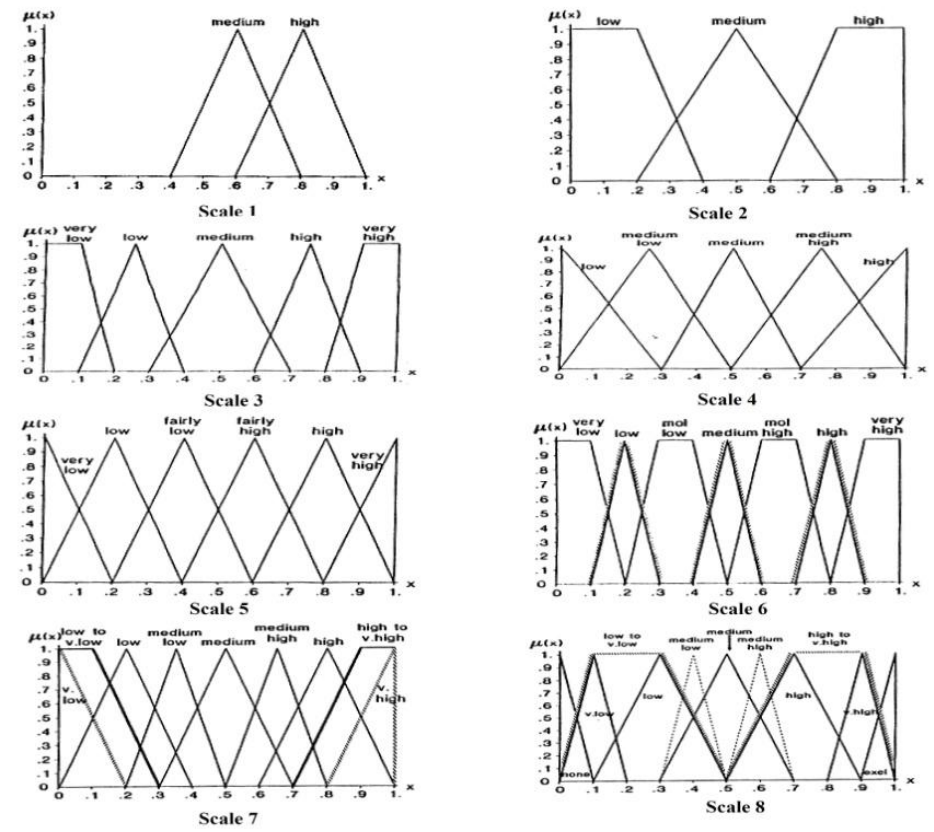

Figure 1. Scale 1-Scale 8 of transformation

\subsubsection{Chen and Hwang Algorithm for solving MADM problems}

This algorithm is described by these steps: 
Step 1: For each fuzzy attribute, the linguistic expressions are first transformed into a fuzzy set.

Step 2: Crisp scores should be assigned to the fuzzy sets using Equations 4,5,6.

Step 3: In this step, a matrix with fuzzy numbers is converted to a matrix with crisp numbers. The alternatives are then ranked by applying any of the MADM methods. In this algorithm, the TOPSIS algorithm is chosen for ranking options.

\section{Conditions for Choosing an Appropriate Fuzzy Ranking Algorithm}

In our previous work (Ramezani and Lu, 2012), we proposed a method for choosing the most appropriate fuzzy ranking algorithm to solve a MADM problem, based on the type and the number of attributes, and the number of alternatives, considering the least computation and time consumption for ranking alternatives. Assume $m$ to be the number of alternatives and $n$ the number of attributes for a MADM problem, and let $J=\left\{\mathrm{j} \mid n_{j}\right.$ is a benefit attribute $\}$ and $J^{\prime}=\left\{\mathrm{j} \mid n_{j}\right.$ is a cost attribute $\}$, then the conditions for choosing the most appropriate fuzzy ranking algorithm to solve this MADM problem are described as follows:

If $(\mathrm{m}>1$ and $\mathrm{n}>1)$ then

If $\left(J=\varnothing\right.$ or $\left.J^{\prime}=\varnothing\right)$ then

SAW algorithm is appropriate

Else

Chen and Hwang algorithm is appropriate

\section{Sensitivity Analysis}

In this section, a sensitivity analysis on the attributes' weight, and a sensitivity analysis on the projects' performance are described.

\subsection{Sensitivity Analysis on the Attributes' Weights}

Sensitivity analysis on the attributes' weight applies the method proposed in (Memariani et al., 2009). Assume in the MADM problem the weights of attributes are normalized, that is:

$$
\sum_{j=1}^{n} \mathrm{w}_{\mathrm{j}}=1
$$

With this assumption, if the weight of one of the attributes changes then the weight of other attributes will change accordingly. If the weight of attribute $p$ th changes to $\Delta_{p}$, then the weight of other attributes change to:

$$
\Delta_{j}=\frac{\Delta_{k} * w_{j}}{w_{k}-1} \quad j=1,2, \ldots, n, j \neq k
$$

In the MADM model of SAW, if the weight of attribute $p$ th changes to $\Delta_{p}$, then the final score of alternative $i$ th would change to $\delta_{i}$, that is:

$$
\delta_{i}=\Delta_{k} * n_{i k}+\sum_{\substack{j=1 \\ j \neq k}}^{n} \frac{\Delta_{k} * w_{j}}{w_{k}-1} * n_{i j} \quad i=1,2, \ldots, m
$$

In addition, in the MADM model of TOPSIS, if the weight of attribute $p$ th changes to $\Delta_{p}$, then the final score of alternative $i$ th would change to:

where:

$$
c l_{i}^{++}=\frac{d_{i}^{+}}{d_{i}^{\prime+}+d_{i}^{\prime-}}
$$

$\gamma=\frac{1-w_{k}-\Delta_{k}}{1-w_{k}}$

$d_{i}^{\prime+}=\left\{\gamma^{2} * d_{i}^{+^{2}}+\left(1-\gamma^{2}\right)\left(v_{i k}-v_{k}^{+}\right)^{2}+\Delta_{k}^{2}\left(n_{i k}-n_{l k}\right)^{2}+2 \Delta_{k}\left(v_{i k}-v_{k}^{+}\right)\left(n_{i k}-n_{l k}\right)\right\}^{1 / 2}$, 
$d_{i}^{\prime-}=\left\{\gamma^{2} * d_{i}^{-2}+\left(1-\gamma^{2}\right)\left(v_{i k}-v_{k}^{-}\right)^{2}+\Delta_{k}^{2}\left(n_{i k}-n_{l^{\prime} k}\right)^{2}+2 \Delta_{k}\left(v_{i k}-v_{k}^{-}\right)\left(n_{i k}-n_{l^{\prime} k}\right)\right\}^{1 / 2}$,

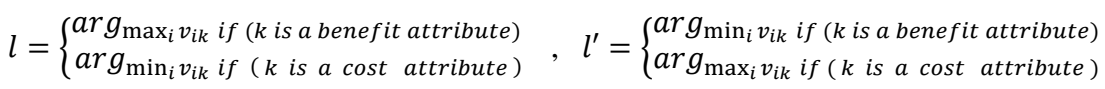

\subsection{Sensitivity Analysis on the Projects' Performance}

Sensitivity analysis on the projects' performance is undertaken with the following proposed equation:

$$
A_{k}=\sum_{j=1}^{n} W_{j} *\left(\sum_{i=1}^{m} \gamma_{i j} * Y_{i}\right)
$$

where $\gamma_{\mathrm{ij}}$ is the score of $i$ th project in $j$ th attribute. $Y_{\mathrm{i}}$ is the project's performance percentage and $W_{\mathrm{j}}$ is the weight of $j$ th attribute. According to equation (22), if the ith project's performance percentage changes, we can determine how the projects' performance will affect promoting the $k$ th goal.

\section{A Conceptual Model for Project Evaluation}

To simulate a decision making process to evaluate projects, all decision making features are considered and a DSS conceptual model is designd as a use case diagram (Figure 2). As can be seen, there are three types of users in the use case diagram: Managing director, Experts (DMs) and Data base supervisor. They follow four fundamental actions:

Task 1. Updating and gathering relevant data: The initial procedure for gathering project data and information includes the following steps:

- Determining the organizations' goals

- Determining criteria (attributes): determining the sets of criteria related to every goal

- Determining sets of projects: classifying projects considering every goals

- Gathering projects' performance data via a control project system

Task 2. Modifying data and information

Task 3. Assessing projects besed on each criterion

Task 4. Sensitivity analysis

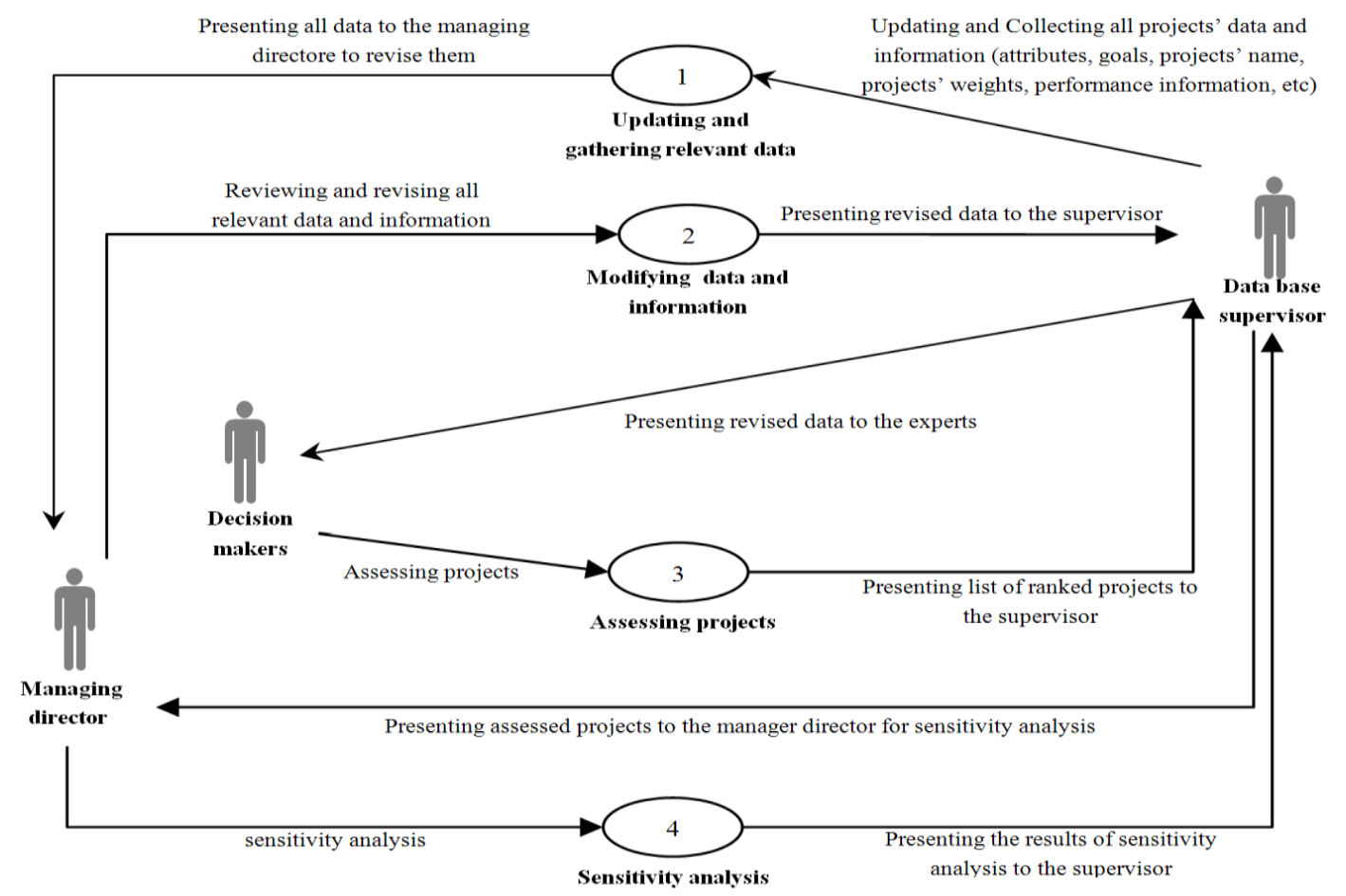

Figure 2. The conceptual model for project performance evaluation (use case diagram) 


\section{A New Project Evaluation Algorithm}

We develop a new project evaluation algorithm on the basis of the proposed FMAGDSS model for project evaluation, which is described as follows:

Step 1: Collecting a first set of inputs data includes: Goals, attributes, alternatives (projects' name), attributes' weights, projects' performance information, and etc.

Step 2: Modifying all projects' data and information.

Step 3: Assessing all projects by considering relevant attributes to determine the score of projects' efficiency in achieving the organization goals: In this step all projects (alternatives) will be assessed by decision makers to determine alternative scores for every attribute $\left[a_{i j}\right](i=1, . ., m$ $j=1, . ., n)$. The results of decision makers' assessment are presented by the linguistic terms of High, Medium High, Medium, Medium Low and Low. To define the membership function of these linguistic terms, the conversion scale 4, which is illustrated in Figure 1, is applied. After collecting all decision makers' opinions, the fuzzy average of all decision makers' opinions is calculated as the group's final assessment, as $\left[A_{i j}\right](i=1, \ldots, m \quad j=1, \ldots, n)$. The results will be used in Step 5 as inputs (second set of inputs) for ranking algorithms to rank alternatives.

Step 4: Choosing the most appropriate fuzzy ranking algorithm: After calculating the group's final decision, the most appropriate fuzzy ranking algorithms for solving the given MADM problem will be chosen based on the conditions that were explained in Section 3.

Step 5: Ranking all projects by the chosen algorithm using the group's final decision: The calculated weight $w_{j}(j=1, . ., n)$ and alternative scores for every attribute $\left[A_{i j}\right](i=1, . ., m j=1, . ., n)$, are applied by the selected fuzzy ranking algorithm as inputs and produce the list of ranked projects as outputs.

Step 6: Final assessment: Evaluation of the projects' importance and its efficiency by: (1) sensitivity analysis of the attributes' weight, and (2) sensitivity analysis on the projects' performance.

Step 7: Choosing the list of the most efficient and important projects to achieve the organization's goals by considering the list of ranked projects and the results of sensitivity analysis. Applying this list, the manager director can choose the most valuable projects, reschedule a projects' time table, focus on important projects and manage investments to enhance the projects' performance.

This algorithm facilitates the decision making process and helps managers to choose the most efficient projects to achieve the organization's goals. Using this algorithm, managers will be able to steer organization resources towards the most inportant projects.

\section{Software Development}

A software has been developed to implement the proposed project performance evaluation algorithm. This software is dynamic to the number and the type of attributes, the number of alternatives and decision makers, and decision making subject, so it is applicable for all decision making environments.

All users of this software should complete their tasks hierarchically. To achive this, the system announces the users via messages that will be sent to them through the "Message Form". In every step of the decision making process, all data will be inserted into a data base. Software interfaces are shown in Fig. 3-6, to explain parts of the decision making process in this research. After the data base supervisor gathers data, these data will be modified by the managing director. Then the system will send a message to all experts (decision makers) to assess projects; the experts will assess projects as shown in Fig. 3. 
They should choose high, medium high, medium, medium low or low to assign a score to every project, cosidering every attribute.
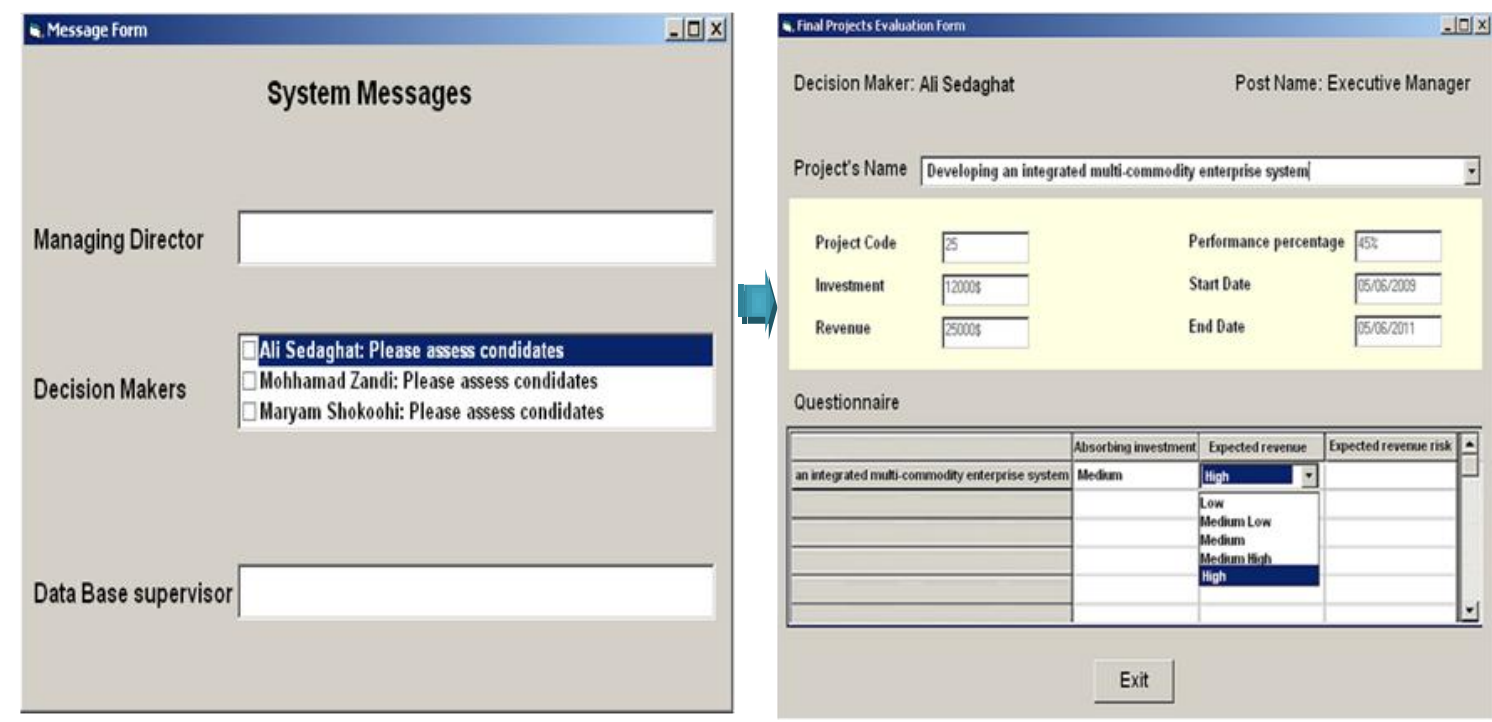

Figure 3. Receiving system message and projects assessing by experts

The system will then evaluate projects via the chosen fuzzy ranking algorithm on the basis of experts' assessment and send a message to the managing director for the final assessment (Fig. 4).

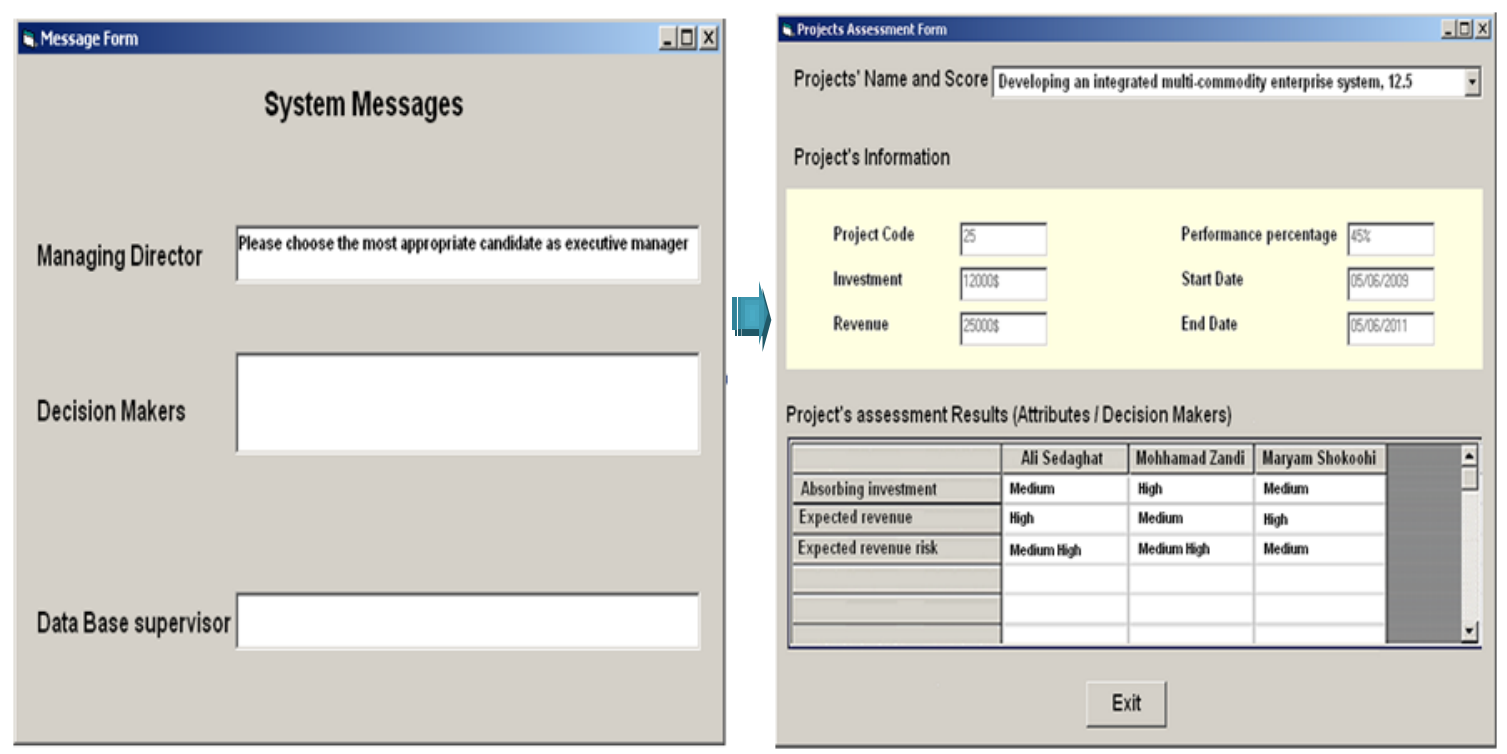

Figure 4. Receiving system message and final assessment by managing director

Finally, to choose the most important and effective projects to achieve the organization's goals, the managing director will apply sensitivity analysis on the "projects' performance" and "criteria weights".

Figure 5 demonstrates how users can apply "sensitivity analysis on projects' performance" . In this section, the user can choose one goal, then choose one of the projects that affects this goal. The user can then change the percentage of the project's performance to see how the project's score for achieving the 
organization's goals, changes. Actually, this part of the software illustrates how the performance of the projects affect the organization goals enhancement.

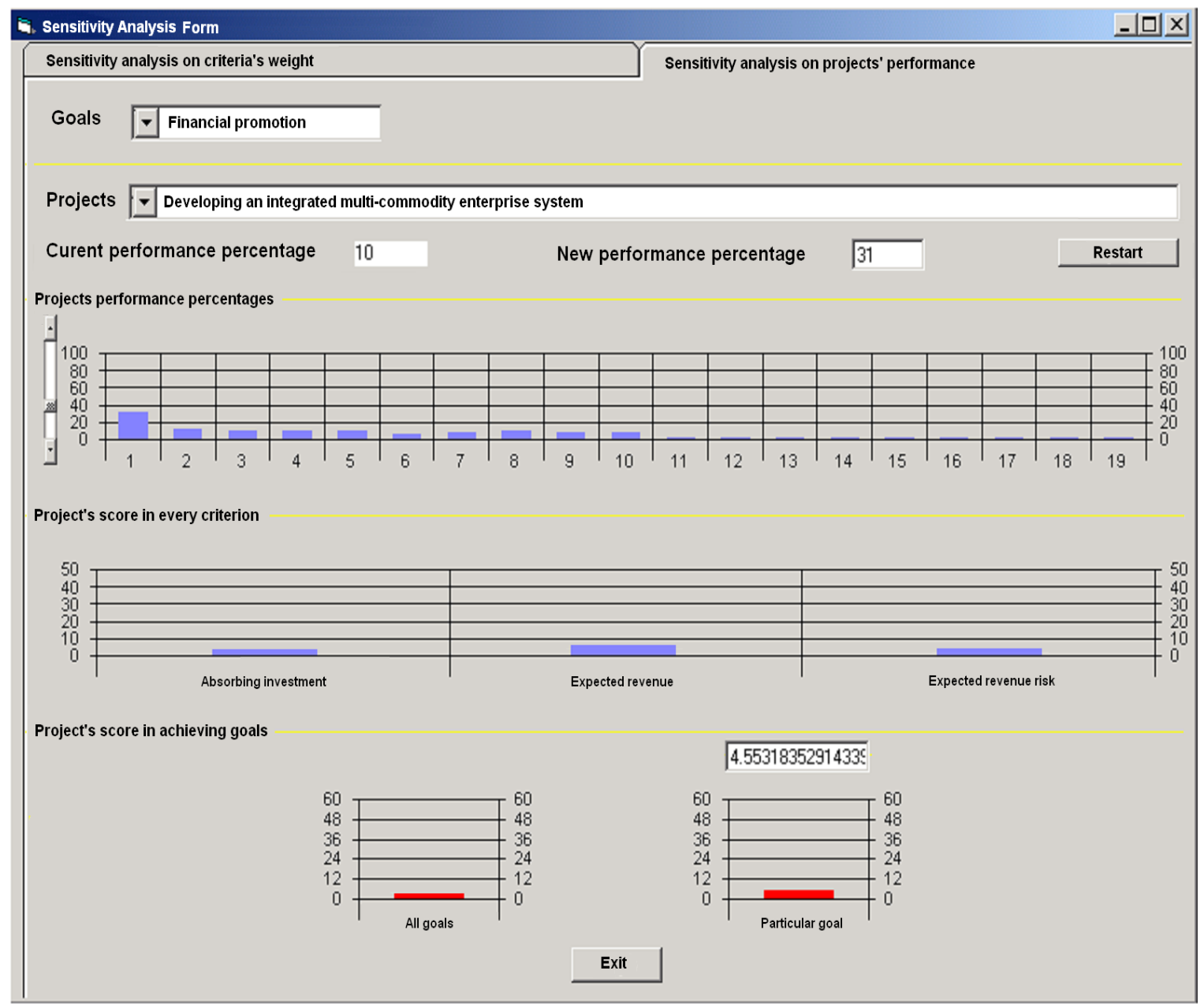

Figure 5. Sensitivity analysis on projects' performance

Figure 6 illustrates how users can apply "sensitivity analysis on ceriteria weights". In this section, the user chooses one criterion, then the software prepares the list of the projects that affect this criterion (related projects), and the name of its related goal. The user then changes the weight of the chosen criterion to see how the following measurements change: (1) the related projects' score in achieving organization goals, (2) related goal achivement rate, and (3) the summation of all organization goal achivement rates. This part of the software illustrates how the different weights of the criteria affect the projects score in achieving organization goals. 


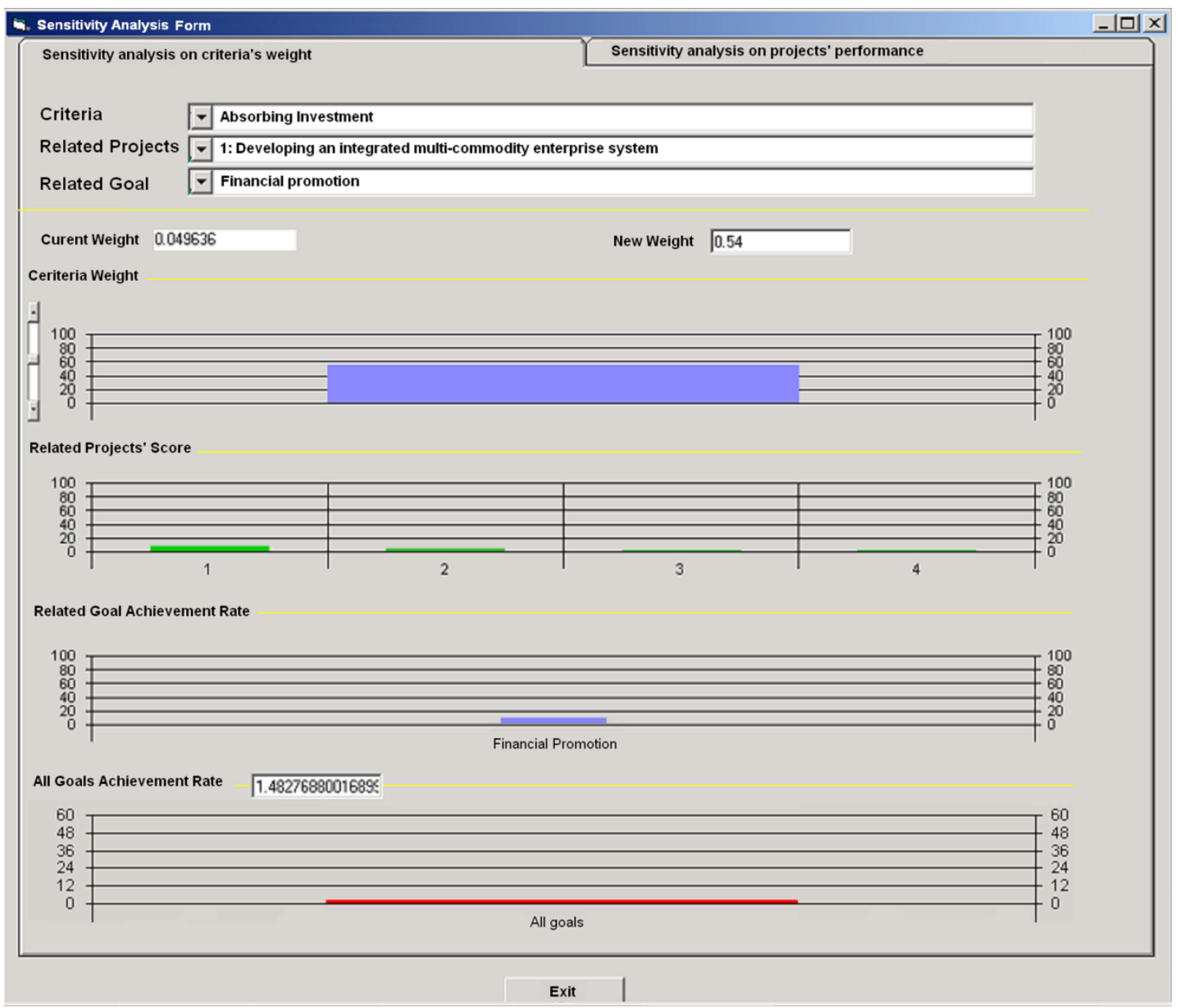

Figure 6. Sensitivity analysis on criteria weights

\section{Conclusion and Future Works}

In regard to the importance of evaluating and choosing the most effective projects to achieve the organizations' goals, which is an FMADM problem with both qualitative and quantitative attributes, this paper has proposed a new FMAGDSS to evaluate projects that has the ability to choose the most appropriate fuzzy ranking method to solve the given problem. In addition, a sensitivity analysis system has been developed concerning the importance of determining the weight of all attributes and the score of alternatives over every attribute, which are obtained through the decision makers' judgement. This sensitivity analysis system assesses the reliability of the decision making process and the impacts of attributes' weights and projects' performance on evaluating projects in achieving an organizations' goals. Finally, we developed a software sysem based on the proposed model. The software is dynamic to the number and type of attributes, the number of alternatives and decision makers, and the decision making subject, so if the decision making environment changes, this software remaisn applicable for the new environment. The proposed model simplifies and accelerates the evaluation process. Applying this model not only helps organizations to choose the most efficient projects for sustainable development, but also helps them to assess the reliability of the decision making process. In our future work we will expand this system to evaluate and rank the project proposals. 


\section{Acknowledgement}

First author would like to thank Professor Azizollah Memariani for bringing this problem to us and helping to implement the solution in a real environment.

\section{References}

BAAS, S. M. \& KWAKERNAAK, H. (1977) Rating and ranking of multiple-aspect alternatives using fuzzy sets. Automatica, 13, 47-58.

BAYKASOGLU, A., GOC KEN , T. \& KAPLANOGLU, V. (2011) A Practical Approach to Prioritize Project Activities Through Fuzzy Ranking. Cybernetics and Systems: An International Journal, 42, 165-179.

BONISSONE, P. P. (1982) A fuzzy sets based linguistic approach: theory and applications. IN GUPTA MM, S. E. (Ed.) Approximate reasoning in decision analysis. Amsterdam North Holland.

BUYUKOZKAN , G. \& RUAN, D. (2008) Evaluation of software development projects using a fuzzy multi-criteria decision approach. Mathematics and Computers in Simulation, 77, 464-475.

CHEN, S.-H. (1985) Ranking fuzzy numbers with maximizing set and minimizing set. Fuzzy sets and Systems, 17, $113-129$.

CHEN, S.-J. J., HWANG, C.-L., BECKMANN, M. J. \& KRELLE, W. (1992) Fuzzy multiple attribute decision making: methods and applications, Springer-Verlag New York, Inc.

CHEN, S.-M. (1988) A new approach to handling fuzzy decision-making problems. Multiple-Valued Logic, 1988., Proceedings of the Eighteenth International Symposium on. IEEE.

CHIANG, T.-A. \& CHE, Z. (2010) A fuzzy robust evaluation model for selecting and ranking NPD projects using Bayesian belief network and weight-restricted DEA. Expert Systems with Applications, 37, 7408-7418.

DE PIANTE HENRIKSEN, A. \& PALOCSAY, S. W. (2008) An excel-based decision support system for scoring and ranking proposed R\&D projects. International Journal of Information Technology \& Decision Making, 7, 529-546.

DEY, P. K. (2006) Integrated project evaluation and selection using multiple-attribute decision-making technique. International Journal of Production Economics, 103, 90-103.

EFSTATHIOU, J. \& RAJKOVIC, V. (1979) Multiattribute decisionmaking using a fuzzy heuristic approach. Systems, Man and Cybernetics, IEEE Transactions on, 9, 326-333.

EFSTATHIOU, J. \& TONG, R. (1982) Ranking fuzzy sets: a decision theoretic approach. IEEE Transactions on System Man and Cybernetics, 12, 655-659.

FISHBURN, P. C. (1967) Additive utilities with incomplete product set: applications to priorities and assignments, Baltimore, MD, ORSA publication.

GOLETSIS, Y., PSARRAS, J. \& SAMOUILIDIS, J.-E. (2003) Project ranking in the Armenian energy sector using a multicriteria method for groups. Annals of Operations Research, 120, 135-157.

HWANG, C.-L. \& YOON, K. (1981) Multiple attribute decision making, Springer.

IMOTO, S., YABUUCHI, Y. \& WATADA, J. (2008) Fuzzy regression model of R\&D project evaluation. Applied Soft Computing, 8, 1266-1273.

JAIN, R. (1976) Decision making in the presence of fuzzy variables. IEEE transactions on Systems, Man and Cybernetics, 6, 698703.

JAIN, R. (1977) A procedure for multiple-aspect decision making using fuzzy sets. International Journal of Systems Science, 8, 1 -7.

KERRE, E. E. (1982) The use of fuzzy set theory in electro cardio logical diagnostics. IN GUPTA MM, S. E. (Ed.) Approximate reasoning in decision analysis. Amsterdam North-Holland

LIANG, Z., YANG, K., SUN, Y., YUAN, J., ZHANG, H. \& ZHANG, Z. (2006) Decision support for choice optimal power generation projects: Fuzzy comprehensive evaluation model based on the electricity market. Energy Policy, 34, 33593364.

LU, J., MA, J., ZHANG, G., ZHU, Y., ZENG, X. \& KOEHL, L. (2011) Theme-based comprehensive evaluation in new product development using fuzzy hierarchical criteria group decision-making method. Industrial Electronics, IEEE Transactions on, 58, 2236-2246.

LU, J., ZHANG, G., RUAN, D. \& WU, F. (2007) Multi-objective group decision making: methods, software and applications with fuzzy set techniques, World Scientific.

LU, J., ZHU, Y., ZENG, X., KOEHL, L., MA, J. \& ZHANG, G. (2009) A linguistic multi-criteria group decision support system for fabric hand evaluation. Fuzzy Optimization and Decision Making, 8, 395-413. 
MA, J., LU, J. \& ZHANG, G. (2010) Decider: A fuzzy multi-criteria group decision support system. Knowledge-Based Systems, 23, 23-31.

MACCRIMMON, K. R. (1968) Decisionmaking among multiple-attribute alternatives: a survey and consolidated approach. DTIC Document.

MAO, Y. \& WU, W. (2011) Fuzzy Real Option Evaluation of Real Estate Project Based on Risk Analysis. Systems Engineering Procedia, 1, 228-235.

MARQUES, G., GOURC, D. \& LAURAS, M. (2011) Multi-criteria performance analysis for decision making in project management. International Journal of Project Management, 29, 1057-1069.

MEMARIANI, A., AMINI , A. \& ALINEZHAD, A. (2009) Sensitivity Analysis of Simple Additive Weighting Method (SAW): The Results of Change in the Weight of One Attribute on the Final Ranking of Alternatives. Journal of Industrial Engineering, 4, 13-18.

RAMEZANI, F. \& LU, J. (2012) A new approach for choosing the most appropriate fuzzy ranking algorithm for solving MADM problems. Autonomous Systems: Developments and Trends. Mallorca Springer-Verlag.

SAGHAEI, A. \& DIDEHKHANI, H. (2011) Developing an integrated model for the evaluation and selection of six sigma projects based on ANFIS and fuzzy goal programming. Expert Systems with Applications, 38, 721-728.

SANNA, U., ATZENI, C. \& SPANU, N. (2008) A fuzzy number ranking in project selection for cultural heritage sites. Journal of Cultural Heritage, 9, 311-316.

WENSTOP, F. (1967) Fuzzy set simulation models in a systems dynamic perspective. Kybernetes 6, 209-218.

ZHANG, G. \& LU, J. (2003) An integrated group decision-making method dealing with fuzzy preferences for alternatives and individual judgments for selection criteria. Group Decision and Negotiation, 12, 501-515. 